\title{
Design, fabrication, and characterization of a solenoid system to generate magnetic field for an ECR proton source
}

\author{
S K JAIN*, P A NAIK and P R HANNURKAR \\ Raja Ramanna Centre for Advanced Technology, Indore 452013 \\ e-mail: skjain@ rrcat.gov.in
}

MS received 3 March 2010; revised 9 June 2010; accepted 13 June 2010

\begin{abstract}
Solenoid coils with iron jacket (electromagnets) have been designed and developed for generation and confinement of the plasma produced by an electron cyclotron resonance source operating at $2450 \mathrm{MHz}$ frequency. The magnetic field configurations designed using the solenoid coils are off-resonance, mirror, and flat, satisfying electron cyclotron resonance condition along the axis of the plasma chamber. 2D Poisson software was used for designing. Details of design, fabrication, and magnetic field mapping of the solenoid coils are presented in this paper.
\end{abstract}

Keywords. ECR plasma source; electromagnet; poisson software.

\section{Introduction}

An electron cyclotron resonance (ECR) plasma source operating at $2450 \mathrm{MHz}$ frequency is under development for extracting $30 \mathrm{~mA}$ proton beam current at $50 \mathrm{keV}$ beam energy. This source will be used as an injector for the $100 \mathrm{MeV}$ proton linac, proposed to be constructed as initial part of a $1 \mathrm{GeV}$ proton synchrotron (Bhawalkar et al 2003). An ECR plasma source finds potential applications in accelerators for producing singly as well as multiply charged ion beams (Bilman et al 1972; Taylor \& Wills 1991; Baskaran et al 1992, 1993). Different magnetic field configurations like off-resonance, resonance, mirror with and without permanent magnets, have been widely used to produce heavy and multiply charged ion beams to confine the plasma axially as well as radially (Alton \& Smith 1994; Drentje 2003; Mishra et al 2004; Koivisto et al 2004; Saitoh et al 2004). In the presence of the magnetic field, as the charged particles are forced to gyrate along the magnetic field lines, their diffusion perpendicular to the magnetic field lines is restricted, thereby confining the plasma radially to produce homogeneous, high-density plasma. ECR proton and deuteron sources (Celona et al 2000, 2004; Gobin et al 2004; Ciavola et al 2004; Delferriere et al 2008) have been widely used for development of high-energy accelerators for transmutations (Carminati et al 1993; Lagniel 1998a, 1998b), neutron spallation (France et al 1996; Lagniel et al 1997), and material science research (Schriber 1994; Tojyo et al 2002; Biri et al 2008). Standard software packages like Poisson and Pandira (http://laacg1.lanl.gov), Opera (www.vectorfields.com),

*For correspondence; Present address: sunil.kumar.jain@cern.ch 
and Intmag (Becker 1989, 1990) are commonly used for simulation of magnetic field patterns using solenoid coils, or permanent magnets. In the present case, the requirement is to get the singly charged ion beam (proton). Accordingly, conventional solenoid coils have been used to produce the ECR plasma, as the solenoid produced magnetic field configurations have been widely used by the ECR community for the extraction of singly charged ion beams.

The '2D Poisson' software package has been used in the present work for simulation of the magnetic field pattern using solenoid coils with iron jacket (electromagnet) for shielding the fringing magnetic field and to reduce the power consumption. The use of jacket is to provide a return path for the magnetic field lines and thereby shield the adjacent components. The use of electromagnet has wide flexibility of tuning the plasma to get the best operating conditions. The use of electromagnet (instead of permanent magnets) helps one to investigate the plasma parameters to optimize the beam current. A conventional ECR ion source uses the principle of adiabatic invariance for mirror reflection, and high magnetic field mode of operation to successfully generate and confine plasma.

\section{Design of the electromagnet}

The magnetic field required to satisfy the ECR resonance condition (Geller 1966; Brown 1989 ; Zhang 1999) is given by $f=2 \cdot 8 B$ (from the electron cyclotron frequency expression $\omega_{c e}=e B / m$, where $f$ is the microwave frequency (in MHz), $B$ is the critical magnetic field (gauss), $m$ is the mass of an electron, and $e$ is the electronic charge. The magnetic field corresponding to a microwave frequency of $2450 \mathrm{MHz}$ is 875 gauss. The magnetic field in the plasma chamber was analysed by using the 2D Poisson software.

To start the simulations, the initial parameters of the solenoid coils with iron jacket were fixed as: inner radius $75 \mathrm{~mm}$, outer radius $150 \mathrm{~mm}$, the gap between solenoid coils $20 \mathrm{~mm}$, width to be $70 \mathrm{~mm}$ and $80 \mathrm{~mm}$ for middle and side solenoid coils respectively. Three solenoid coils were used to get; (i) mirror field, (ii) flat field, and (iii) off-resonance field configuration. Two side solenoid coils, which were identical, generated the mirror field, and the middle solenoid coil was used to compensate the dip in the magnetic field, to have a flat magnetic field. The use of flat field configuration with high field in the centre has been reported to provide better extraction of proton current in the high intensity light ion sources (Celona et al 2000, 2004; Gobin et al 2004; Ciavola et al 2004; Delferriere et al 2008). The combinations of these three solenoid coils can also produce the off-resonance magnetic field. The permeability table for soft iron, low carbon, TATA 'A' grade steel has been incorporated in the Poisson code itself. A number of iterations were carried out to get the desired magnetic field configurations. This was accomplished by varying the size of the solenoid coils, thickness of iron jacket, and amp-turns (NI). The optimum values of NI for the side and middle solenoid coils were obtained to be 12950 and 8250 respectively. Based on these parameters, the total length of the conductor was calculated. A copper conductor having a square cross-section of $5 \mathrm{~mm} \times 5 \mathrm{~mm}$ with hole diameter $3 \mathrm{~mm}$ for water cooling was used. The optimized design parameters for solenoid coil with iron jacket are presented in table 1 . The optimized axial flux along the axis of the source is shown in figure 1. The optimized magnetic field profiles using Poisson software for (a) mirror magnetic field, and (b) flat magnetic field are shown in figure 2. The calculation was also performed analytically (Montgomery 1966) using standard relations for calculating magnetic field. 
Table 1. The optimized design parameters for solenoid coil with iron jacket.

\begin{tabular}{|c|c|c|c|}
\hline Description & Solenoid coil 1 & Solenoid coil 2 & Solenoid coil 3 \\
\hline Coil type & \multicolumn{3}{|c|}{ Water cooled solenoid coils } \\
\hline Coil size & $\phi 300 \mathrm{~mm} \times 80 \mathrm{~mm}$ & $\phi 300 \mathrm{~mm} \times 70 \mathrm{~mm}$ & $\phi 300 \mathrm{~mm} \times 80 \mathrm{~mm}$ \\
\hline $\begin{array}{l}\text { Bore diameter } \\
\text { amp-turns (NI) }\end{array}$ & 12950 & $\begin{array}{l}\phi 150 \\
8250\end{array}$ & 12950 \\
\hline Conductor size & \multicolumn{3}{|c|}{ Rectangular with cross-section $5 \mathrm{~mm} \times 5 \mathrm{~mm} \times$ hole diameter $3 \mathrm{~mm}$} \\
\hline $\mathrm{N}, \mathrm{N} / \mathrm{L}, \mathrm{L}$ & $144,12,12$ & $110,10,11$ & $144,12,122$ \\
\hline Total conductors length & $95 \mathrm{~m}$ & $80 \mathrm{~m}$ & $95 \mathrm{~m}$ \\
\hline Power supply & \multicolumn{3}{|c|}{ 0-32 V, 100 A dc (Three) } \\
\hline Coil resistance (measured) & $0 \cdot 146 \mathrm{ohms}$ & $0.127 \mathrm{ohms}$ & $0 \cdot 156 \mathrm{ohms}$ \\
\hline
\end{tabular}

\section{Fabrication of the electromagnet}

The solenoid coils were fabricated using super enameled hollow copper conductor (refrigeration type) of square cross-section $(5 \mathrm{~mm} \times 5 \mathrm{~mm}$ ) with hole diameter $3 \mathrm{~mm}$ (for water circulation). The insulation to the conductor layer was provided using ' $\mathrm{H}$ ' class fibreglass insulation tape. The diameter of the bore was $150 \mathrm{~mm}$ so that plasma chamber including water-cooling jacket and high voltage insulator could be fitted in this. One side flange of the plasma chamber was split type, so that the solenoid coils could be fitted to the plasma chamber. The bore of the solenoid coils was fabricated using high voltage glass epoxy. Each side solenoid coil had 12 turns/layer (N/L), and 12 layers (L) \{i.e. 144 turns (N)\}, and the central solenoid coil had 10 turns/layer (N/L) and 11 layers (L) \{i.e. 110 turns (N)\}. The total length of the conductor used for the side solenoid coils and the central solenoid coil was 95 meters and 80 meters respectively. The coils were impregnated into high voltage, high temperature epoxy for the outer layer insulation. The iron jacket of the solenoid coils was fabricated from $10 \mathrm{~mm}$ thick low carbon TATA 'A' grade steel. The jacket was fabricated in five parts consisting of two side plates and three cylindrical shapes, of diameter equal to the

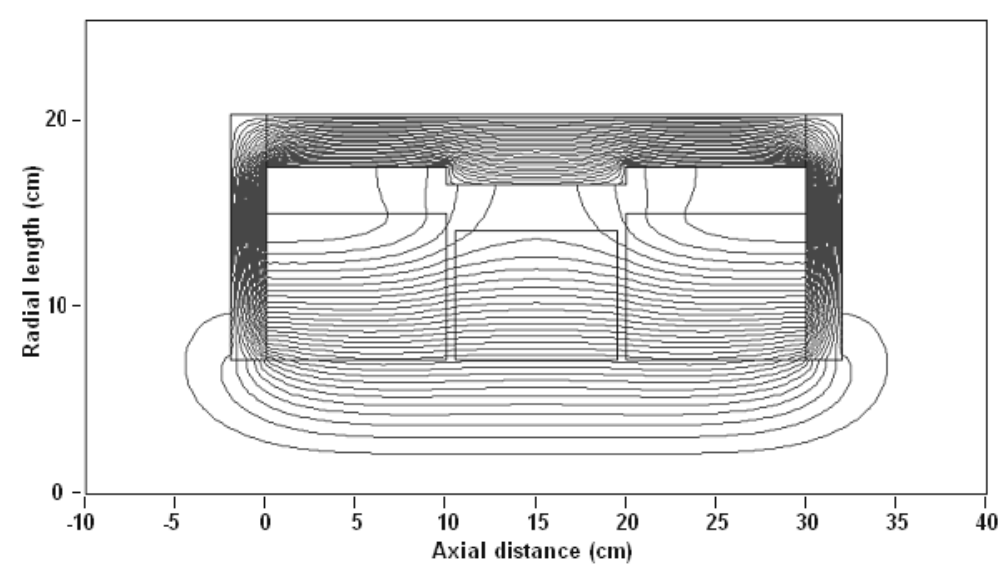

Figure 1. The optimized axial flux along the axis of the source. 

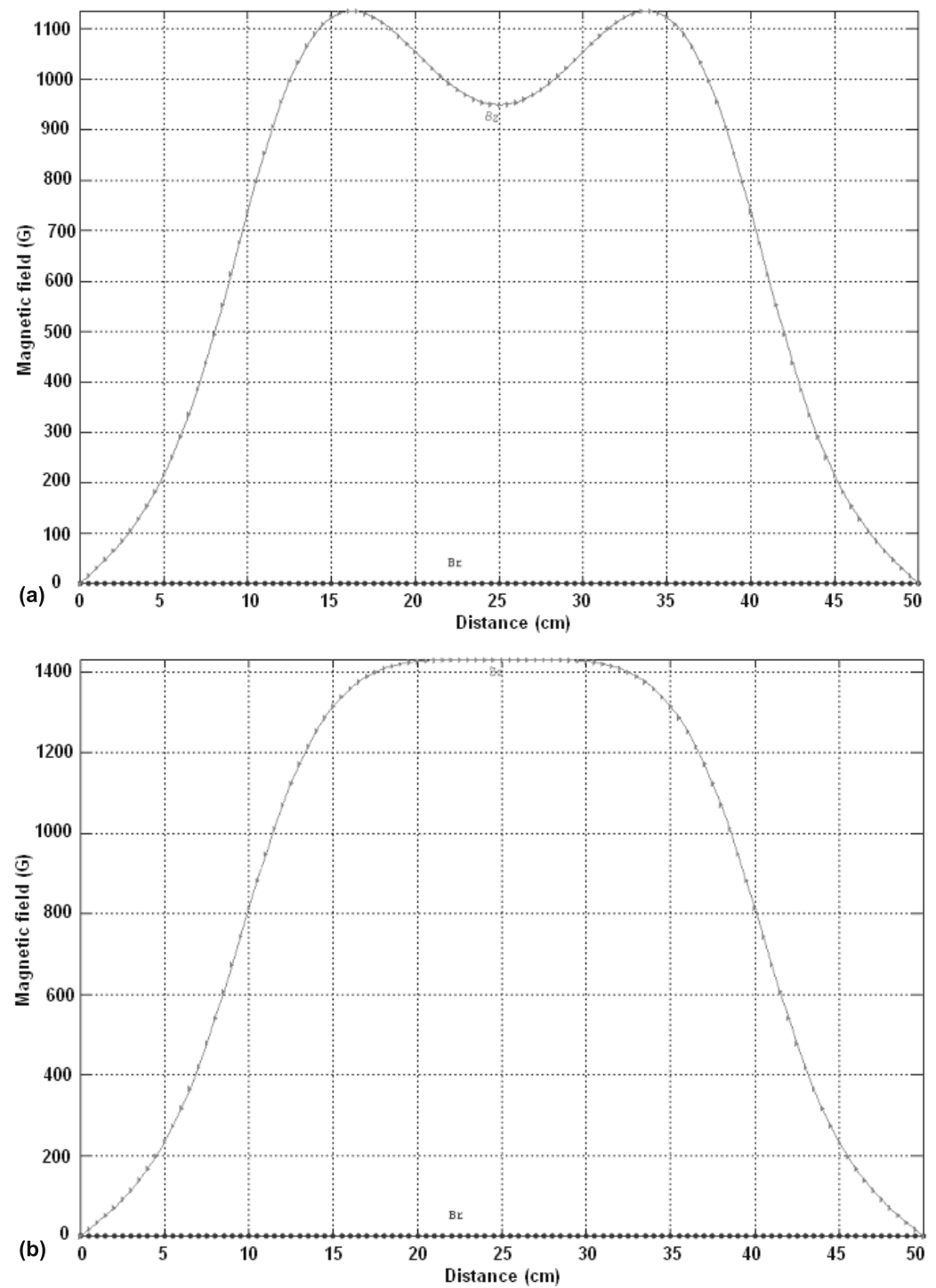

Figure 2. The optimized magnetic field profiles using Poisson software for (a) mirror magnetic field, and (b) flat magnetic field.

bore diameter of the solenoid coils. These electromagnets were placed around the plasma chamber to produce the necessary magnetic field. The cross-sectional view of the integrated assembly of the three-solenoid coils with iron jacket (electromagnet) is shown in figure 3.

The solenoid coils were cooled using low conductivity water (conductivity less than $1 \mu \mathrm{S} / \mathrm{cm}$ ) having inlet temperature of $27^{\circ} \mathrm{C}$. Based on the length of the conductor, water pressure drop and flow, the inlet and outlet connections were provided. Total five inlet and five outlet connections were provided. The water flow rate 3 liter $/ \mathrm{min}$. and pressure $3.5 \mathrm{~kg} / \mathrm{cm}^{2}$ was maintained. The rise in temperature was restricted to less than $20^{\circ} \mathrm{C}$. The inductance and resistance of the solenoid coils were measured using precision LCR meter (Model:PM6306, 


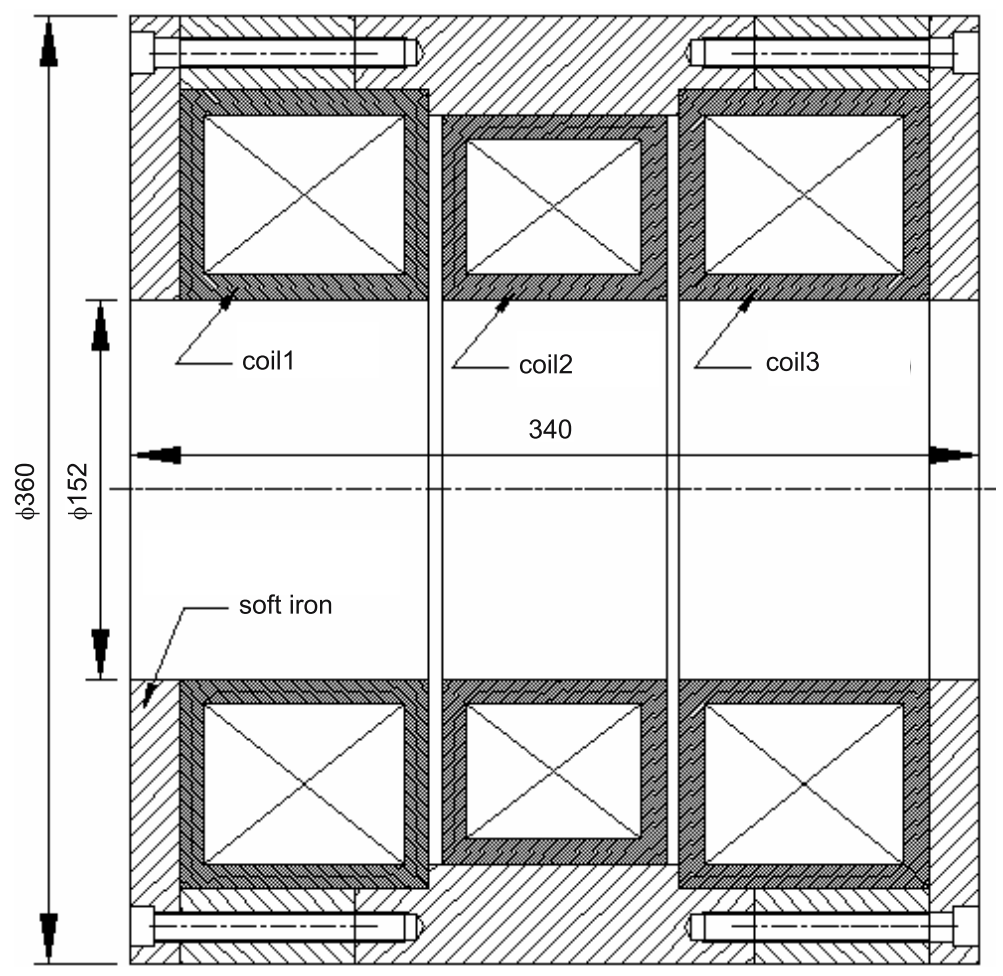

Figure 3. The cross-sectional view of the integrated assembly of the three-solenoid coils with iron jacket (electromagnet).

Make:Fluke). The high voltage insulation to the solenoid coils up to $5 \mathrm{kV}$ dc was tested using high voltage Megger (Model:220123-47, Make:Megger, Biddle, England).

\section{Magnetic field mapping}

The performance of an electromagnet gets influenced by the design and material limitations, errors in construction, and the stability of the power supply used to energize it. Therefore, after the electromagnets are built, they have to be tested for their performance. The magnetic field measurements were done using computer controlled three-axis coordinate measuring machine (Jain et al 2010). A Hall probe (MPT-141) was attached at the Y-arm with a probe holder. The Hall probe had field ranges of $0 \cdot 3,0 \cdot 6,1 \cdot 2,3 \cdot 0$ tesla and corresponding serial/general purpose interface bus (GPIB) resolutions $0 \cdot 001,0.01,0.01,0.01 \mathrm{G}$, respectively. The probe size was $15 \mathrm{~mm} \times 5 \mathrm{~mm} \times 2 \mathrm{~mm}$, with sensitive area $1 \mathrm{~mm} \times 0.5 \mathrm{~mm}$. Measurement rate was 10 measurements per second. The error associated in the magnetic field measurements was less than $0.5 \%$. The electromagnets were energized using three independent dc power supplies of rating $0-32 \mathrm{~V}$ and $100 \mathrm{~A}$. The stability of the power supplies was $0 \cdot 1 \%$.

The quality of the electromagnets was fully characterized before installation in the dynamical environment. The successful operation of an ECR source greatly depends on the quality of the magnetic elements and uniformity in the magnetic field (better than $5 \mathrm{G}$ ). Imperfections in 

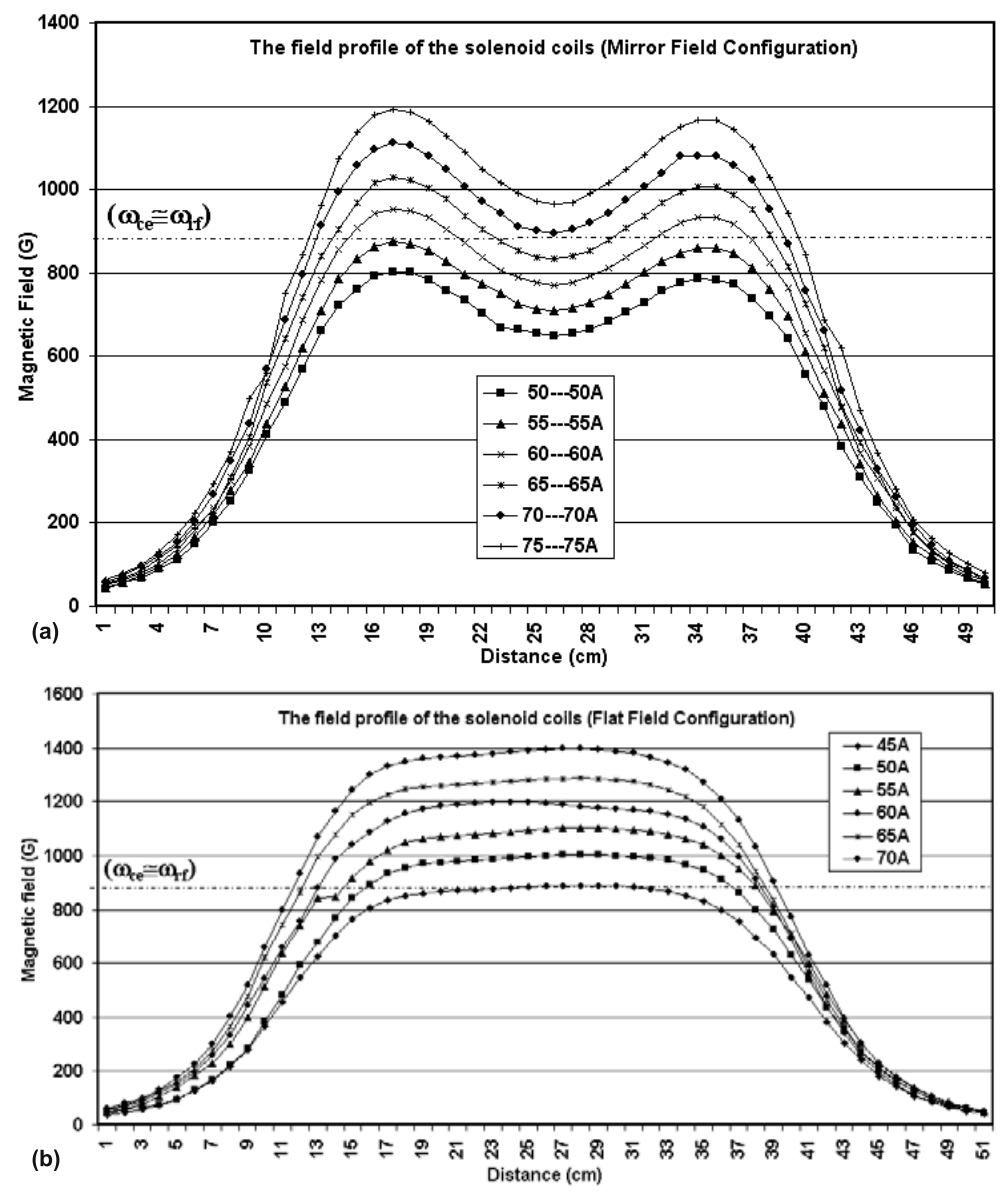

Figure 4. The measured magnetic field profiles along the axial distance for (a) mirror magnetic field, and (b) flat magnetic field.

the magnetic field can cause diffusion of the plasma particles to the wall of the plasma chamber. The measured magnetic field profiles along the axial distance for (a) mirror magnetic field, and (b) flat magnetic field are shown in figure 4. The measured values (i.e. experimental data shown in figure 4 ) and the design values (i.e. simulation results shown in figure 2) agree within $3 \%$. The electromagnets were characterized for $45 \mathrm{~A}$ to $75 \mathrm{~A}$ at steps of $5 \mathrm{~A}$. The variation of magnetic field with solenoid current is shown in figure 5. It is observed to be linear with a slope of $21.5 \mathrm{G} / \mathrm{A}$. The ampere-turns were determined to produce the maximum possible magnetic field on the axis. The size and the power consumption of the solenoid coils were the limiting factors. In order to increase the axial magnetic field and to obtain the required distribution, soft iron jacket were used. The mirror ratio (maximum to minimum) field of the electromagnet was $1 \cdot 1$. A maximum flat field of $1400 \mathrm{G}$ was obtained when all the three coils were independently energized. Using these magnets, ECR plasma was successfully produced (Jain et al 2007) using nitrogen, argon, and hydrogen gases. The plasma was characterized using a Langmuir probe (Jain et al 2006). A total ion beam current of $2.5 \mathrm{~mA}$ was extracted, with two-electrode extraction geometry, at $15 \mathrm{keV}$ beam energy. 


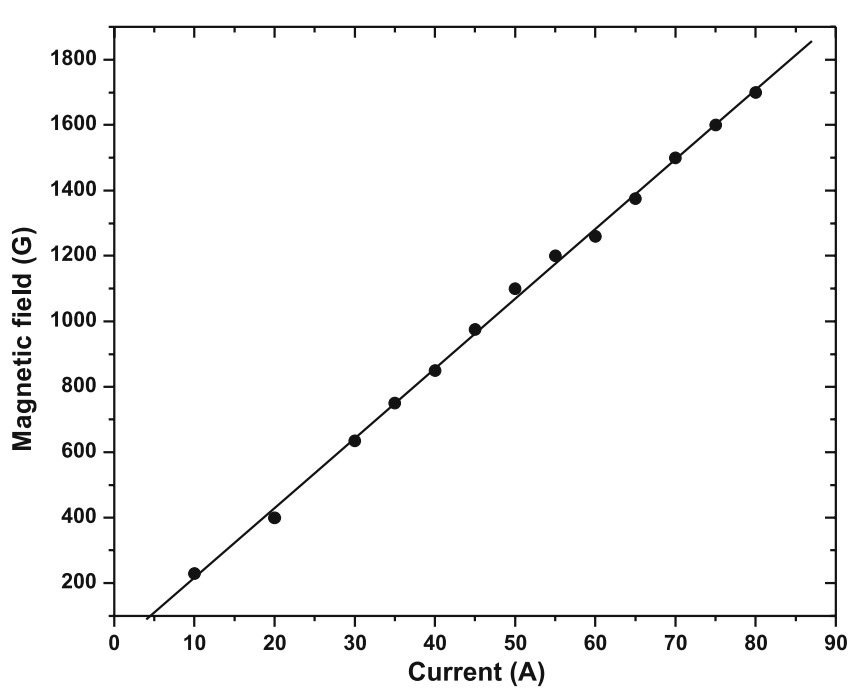

Figure 5. The variation of the magnetic field with solenoid current.

\section{Conclusions}

The solenoid coil magnets described here offers a continuous control of the axial magnetic field, giving tuning capability. The fine tuning the magnetic field is very important for the stability of an ECR plasma source, as any small change in the distribution of the axial magnetic field can results large changes in the source parameters. The three solenoid coil system is capable of producing off-resonance, mirror, and flat magnetic field configurations. A mirror ratio of 1.1 and a maximum flat field of $1400 \mathrm{G}$ has been obtained. The measured values and the design values are found to have a good agreement within $3 \%$.

\section{References}

Alton G D, Smith D N 1994 Design studies for an advanced ECR ion sources. Rev. Sci. Instrum. 65: 775-787

Baskaran R, Jain S K, Ramamurthy S S 1992 E-plane horn excitation of slow wave structures for obtaining high-density electron cyclotron resonance plasmas. Rev. Sci. Instrum. 63(3): 1939-1944

Baskaran R, Heurtier J M, Hill C E 1993 Modelling of the electron cyclotron resonance sulphur source. Rev. Sci. Instrum. 64(1): 191-196

Becker R 1989 Intmag: A program for the calculation of magnetic fields by integration. Nucl. Instr. Meth. Phys. Res. B42(3): 303-306

Becker R 1990 Magnetic fields calculated by Intmag compared with analytical solutions and precision measurements. Nucl. Instr. Meth. Phys. Res. A298: 13-21

Bhawalkar D D, Bhujle A G, Fatnani P, Hannurkar P R, Joshi S C, Karmarkar M G, Kotaiah S, Mhaskar S P, Pande S A, Prabhu S S, Shinde R S, Shukla S K, Singh G 2003 Indian Spallation Neutron Source. InPac-03: 57-60

Bilman S, Geller R, Hess W, Jacquot B C 1972 A high intensity ECR stripped ion source. IEEE Trans. Nucl. Sci. NS-19(2): 200-203

Biri S, Ivan I, Juhasz Z, Sulik B, Hegedus C, Jenei A, Kokenyesi S, Palinkas J 2008 Application of the Atomki-ECRIS for materials research and prospects of the medical utilization. ECRIS-08 Chicago IL USA

Brown I G 1989 The physics and technology of ion sources (New York: John Willey \& Sons) 
Carminati F, Kalapish Z R, Revol J P, Roche C, Rubio J A, Rubbia C 1993 An energy amplifier for cleaner and inexhaustible nuclear energy production driven by particle beam accelerator. Report No: CERN/AT/93-47

Celona L, Ciavola G, Gammino S, Gobin R, Ferdinand R 2000 Trips: The high intensity proton source for the Trasco project. Rev. Sci. Instrum. 71(2): 771-773

Celona L, Ciavola G, Gammino S, Chines F, Presti M, Ando L, Guo X H, Gobin R, Ferdinand R 2004 Status of the Trasco intense proton source and emittance measurements. Rev. Sci. Instrum. 75(5): $1423-1426$

Ciavola G, Celona L, Gammino S, Presti M, Ando L, Passarello S, Zhang X Z h, Consoli F, Chines F, Percolla C, Calzona V, Winkler M 2004 A version of the Trasco intense proton source optimized for accelerator driven system purposes. Rev. Sci. Instrum. 75(5): 1453-1456

Delferriere O, Menezes D De, Gobin R, Harrault F, Tuske O 2008 Electron cyclotron resonance $140 \mathrm{~mA}$ D+ beam extraction optimization for IFMIF EVEDA accelerator. Rev. Sci. Instrum. 79: 02B723-1-3

Drentje A G 2003 Techniques and mechanisms applied in electron cyclotron resonance sources for highly charged ions. Rev. Sci. Instrum. 74(5): 2631-2645

France A, Gobin R, Delferriere O, Leroy P A, Delaunay M, Farchi A 1996 High current proton and deuteron ECR source developments at CEA. EPAC-96 Sitges Barcelona, Spain

Geller R 1966 Electron cyclotron resonance ion sources and ECR plasmas. (London: Institute of Physics)

Gobin R, Beauvais P Y, Bogard D, Charruau G, Delferriere O, Menezes D De, France A, Ferdinand R, Gauthier Y, Harrault F, Mattei P, Benmeziane K, Leherissier P, Paquet J Y, Ausset P et al 2004 Status of the light ion source development at CEA/Saclay. Rev. Sci. Instrum. 75(5): 1414-1416 http://laacg1.lanl.gov Poisson code, Reference manual, LA-UR-87-126, LANL 1987

Jain S K, Jain A, Sharma D, Hannurkar P R 2006 Acquisition and analysis of Langmuir probe characterization for ECR plasma. Indian J. Phys. 80: 1011-1015

Jain S K, Jain A, Hannurkar P R, Kotaiah S 2007 Characterization of plasma parameter, first beam results, and status of electron cyclotron resonance source. Rev. Sci. Instrum. 78: 053301-1-6

Jain S K, Malik Ritesh, Sekar K, Naik P A, Hannurkar P R 2010 Design, fabrication and measurement of 90-degree mass-analyzing magnet. Indian J. Pure and Applied Phys. 48: 315-320

Koivisto H, Suominen P, Tarvainen O, Hitz D 2004 A modified permanent magnet structure for a stronger multipole magnetic field. Rev. Sci. Instrum. 75(5): 1479-1481

Lagniel J M 1998a Proc. of 19th International Linac Conference Chicago IL

Lagniel J M 1998b A review of Linacs and beam transport systems for transmutation. EPAC-98 Stockholm Sweden

Lagniel L M, Joly S, Lemaire J L, Mueller A C 1997 IPHI, The Saclay high-intensity proton injector project. PAC-97 Vancouver Canada

Mishra L N, Shibata K, Ito H, Yugami N, Nishida Y 2004 Characteristics of electron cyclotron resonance plasma generated in a rectangular waveguide by high power microwave. Rev. Sci. Instrum. 75(1): 84-89

Montgomery D B 1966 Solenoid Magnet Design. (New York: John Wiley \& Sons)

Saitoh Y, Ohlwshi K, Arakawa K 2004 Development of $13 \mathrm{GHz}$ compact electron cyclotron resonance ion source. Rev. Sci. Instrum. 75(5): 1502-1505

Schriber S O 1994 Survey of proposed high intensity accelerators and their applications. EPAC-94 London UK

Taylor T, Wills J S C 1991 A high current low-emittance dc ECR proton source. Nucl. Instr. Meth. Phys. Res. A309: 37-42

Tojyo E, Katayama I K, Jeong S C, Oyaizu M, Ishiyama H, Kawakami H, Enomoto K, Miyatake H 2002 A compact 2.45 ECR ion source with permanent magnets for material science. Rev. Sci. Instrum. 73(2): 586-588 www.vectorfields.com Opera 3D, (c) Vector Field Limited, Oxford

Zhang H 1999 Ion Sources. (New York: Springer) 